\title{
Las construcciones verbo-nominales en la enseñanza de ELE
}

\section{Verb-Noun Constructions in SFL learning}

\author{
Xavier Pascual-López \\ Uniwersytet im. Adama Mickiewicza w Poznaniu (Polonia) \\ xavpas@amu.edu.pl
}

\begin{abstract}
This paper proposes an approach to verb-noun constructions that allows them to be understood holistically, which facilitates the acquisition of their distinctive features (for example, the presence or absence of an article) and their effective correlation with certain communicative functions and situations. Therefore, their use will not only be lexically and grammatically appropriate, but the apprentices will be able to make use of them as a whole acquired and associated with a certain communicative situation or function. As a result, their fluency will gain naturalness and will be closer to native.
\end{abstract}

Keywords: Verb-Noun Constructions, collocations, SFL learning, Spanish articles, Holistic Approach to Learning

\section{INTRODUCCIÓN}

En la didáctica de una segunda lengua (L2) se acepta la necesidad de integrar la gramática, la perspectiva comunicativa y las cuatro destrezas lingüísticas, con el claro objetivo de dotar al discente de una competencia comunicativa que le permita desenvolverse, sin graves incidentes, en la comunidad meta. Sin embargo, en el caso del español como lengua extranjera (ELE), uno de los escollos que todavía perduran es la inclusión del léxico en la programación de los diferentes niveles de enseñanza-aprendizaje, debido a la dificultad de su planificación (especialmente en niveles medios y avanzados). Como indica Navarro (2003, p. 103), “el dominio léxico, en realidad, se 
ejercita en los cuatro componentes de la competencia comunicativa", de modo que el léxico no puede desvincularse de su cotexto (es decir, de las coocurrencias sintácticas de las diferentes unidades léxicas) y de su finalidad comunicativa.

El objetivo de este artículo es concienciar de la importancia de la enseñanza holística de una serie de construcciones que llamamos verbo-nominales, con las cuales se pueden expresar determinadas funciones comunicativas y de cuyo aprendizaje holístico depende no solo la corrección lingüística, sino también su adecuación pragmática y la consecución de una fluidez similar a la nativa. Para ejemplificarlo, se analizará cómo estas construcciones aparecen un manual actual de ELE.

\section{CONSTRUCCIONES VERBO-NOMINALES: ¿FRASEOLOGÍA O SINTAXIS LIBRE?}

A menudo se insiste en la necesidad de incorporar la fraseología en los contenidos curriculares de toda L2, ya que en los aprendices suele advertirse una "carencia de expresiones idiomáticas y locuciones y el mal uso de expresiones pragmáticas y colocaciones" (Solano Rodríguez, 2007, p. 201), lo cual aleja el discurso de un aprendiz del de un nativo. Otra cuestión es, no obstante, en qué nivel deben introducirse, pues para muchos su aplicación en un nivel inicial es inapropiada ${ }^{1}$. Parece evidente que estas voces responden a una concepción estrecha de la fraseología que la circunscribe a las locuciones o a las unidades idiomáticas ${ }^{2}$. No hay que olvidar que la idiomaticidad está sometida a gradación, por lo que hay fraseologismos de una mayor transparencia semántica y que, además, pueden resultar apropiados a los objetivos comunicativos de un nivel A1 o A2. Por otro lado, parece arriesgado defender que no deben incorporarse unidades fraseológicas en estos niveles, puesto que el alumnado se enfrenta a ellas desde que oye y aprende a usar fórmulas rituales como ;Buenos días! o lee $h a z$ una lista en el enunciado de un ejercicio.

En torno a esta tendencia a limitar la fraseología a las unidades idiomáticas, Howarth (1998a, 1998b) considera que la adquisición de estas unidades es la que crea menos problemas, dado que su grado de fijación y su peculiaridad semántica hacen que resulten más perceptibles y llamativas para docentes y discentes. De acuerdo con este autor, la didáctica de la fraseología en una L2 adolece de un evidente hándicap:

\footnotetext{
${ }^{1}$ Véanse, por ejemplo, estudios pioneros como los de Ruiz Campillo \& Roldán Vendrell (1993), Forment (1998a, 1998b), García Muruais (1998), Ruiz Gurillo (2000), Dante Hernández (2003).

${ }^{2}$ De hecho, bien conocida es la controversia entorno al alcance de la fraseología, que oscila entre dos concepciones: la amplia incluye unidades sintácticamente a nivel oracional o que constituyen enunciado por sí mismas, mientras que la estrecha suele limitar su objeto de estudio a unidades cuyos límites se ajustan a los de la palabra o el sintagma; además, no falta quien excluye las colocaciones también de esta última concepción, por no considerarlas un fenómeno fraseológico, sino un caso de selección léxica, situándolas en la interfaz léxico-sintaxis (Bosque, 2001a, p. 10).
} 
en un nivel avanzado se ha llegado a capacitar al aprendiz para manejar los dos extremos del continuo de la variabilidad (las combinaciones libres y las unidades idiomáticas), pero "many learners fail to understand the existence of the central area of the phraseological spectrum [...]. It is in handling restricted collocations that errors of both a lexical and grammatical nature constantly occur" (1998b, p. 186). En el ámbito de ELE fue Higueras García (1997) quien destacó la relevancia de lo que entonces llamó combinación sintagmática: "al utilizar un concepto más amplio que la unidad palabra, ayudamos al alumno a combinarlas y favorecemos de este modo el proceso de aprendizaje y recuperación y mejoramos su competencia comunicativa" (p. 46). La autora desarrolla estos argumentos en su monografía sobre didáctica de las colocaciones, llegando a la conclusión lógica de que las que deben enseñarse son las más usuales, centrándose en las que tengan una cohesión media, dado que el aprendiz suele conocer las palabras pero desconocer su capacidad de combinación (2006, pp. 72-77).

Sin embargo, el propio concepto de colocación puede resultar un poco coercitivo cuando se aplica a la didáctica de una L2. El aprendiz debe ser capaz de crear un mapa mental de conexiones entre unidades léxicas que le permitan combinarlas de forma fluida, de modo que a la hora de aprender una L2 pueden resultar superfluas diferencias terminológicas entre colocaciones propiamente dichas (p. ej. hacer/practicar deporte) y otras combinaciones que se corresponden con las solidaridades léxicas de Coseriu (1991) (p. ej. escribir/leer un texto ${ }^{3}$ ). Es evidente que estas combinaciones semánticas a menudo son universales, responden a relaciones extralingüísticas y no generan problemas más allá de conocer las palabras, pero al mismo tiempo hay que tener en cuenta que la capacidad combinatoria de una unidad léxica incluye también este tipo de combinación. Pongamos como ejemplo los verbos que pueden coaparecer con frecuencia junto al sustantivo pelicula: ver, mirar, hacer, realizar, rodar, filmar, poner, pasar, proyectar, subir, bajar, descargar, dirigir, protagonizar, salir en, aparecer en, participar en ${ }^{4}$. Este conjunto de verbos puede considerarse parte del contorno lingüístico en el que el sustantivo en cuestión aparece como objeto, atendiendo a diversos criterios lingüísticos (diferencias diatópicas como ver/mirar, diafásicas como poner/proyectar o semánticas como dirigir/protagonizar) y, por tanto, son los verbos con los que un aprendiz de ELE debería asociarlo en su interlengua durante su aprendizaje. Que algunas de estas combinaciones puedan considerarse colocaciones (por ej. con verbos soporte como hacer) o solidaridades léxicas (con verbos específicos del campo semántico en cuestión como filmar) es algo que resulta hasta cierto

${ }^{3}$ En este tipo de combinación, la selección de una palabra no es arbitraria (como lo sería la elección del colocativo en el caso de las colocaciones), sino que una unidad léxica aparece semánticamente determinada por la otra, pues en el sentido de una está implícito el de la otra, lo cual favorece su coaparición. Usando el ejemplo que proporcionamos, en el sentido de escribir o leer está incluido el sema texto, por lo que es normal que dichos verbos se combinen con sustantivos que también lo contengan: texto, libro, novela, articulo, noticia, etc.

${ }^{4}$ Los verbos propuestos se basan en las concordancias encontradas en el corpus CORPES XXI de la RAE. 
punto superfluo para el proceso de enseñanza-aprendizaje, por más que la arbitrariedad o no motivación de algunas colocaciones sea un factor de dificultad añadido a dicho proceso.

Quizás por ello, a la hora de aplicarse a la didáctica, puede ser de mayor interés el concepto formulaicidad (Wray, 2002, pp. 4-10), cuyo enfoque es mucho más aplicado que el de la propia fraseología, a menudo demasiado teórica o taxonómica. Desde esta perspectiva, se habla de lenguaje formulario o secuencias formularias como "a sequence, continuous or discontinous, of words or other meaning elements, which is, or appears to be, prefabricated: that is, stored and retrieved whole from memory at the time of use, rather than being subject to generation or analysis by the language grammar" (Wray, Perkins, 2000, p. 1).

Según esta concepción, la lengua dispone de unos esquemas estructurales que constituyen un armazón sobre el que se sustenta una ejecución lingüística fluida, cuya variedad dependerá más de la elección de las unidades léxicas que de la propia estructura. Pawley \& Syder (2000) ponen en duda que se pueda producir un mensaje espontáneo, fluido y coherente con estructuras sintácticas complejas sin recurrir a combinaciones léxicas prefabricadas. A su vez, Wray \& Perkins (2000) consideran que las secuencias formularias no requieren que se generen de forma analítica o composicional, sino que se usan e interpretan de forma holística, con lo que la expresión puede ser más fluida ${ }^{5}$.

Volviendo a nuestro ejemplo con el sustantivo película, la creación de un mapa mental que permita asociarlo con esa serie de verbos (o verbos con preposición) hará que el aprendiz construya un discurso más fluido, con una unidad léxica atrayendo a la otra y percibiéndose construcciones como rodar una película de forma holística, como un todo. En realidad, nuestra perspectiva se acerca a la gramática de la construcción, según la cual los patrones lingüísticos se almacenan como una unión en la que forma, sentido o función discursiva están fusionados debido a su frecuencia de coaparición (Goldberg, 2006, p. 5)

Por todo ello, en nuestro análisis preferimos hablar de construcciones verbonominales y el corpus que hemos elaborado a partir de los manuales puede resultar variopinto desde una perspectiva puramente fraseológica. Las construcciones analizadas pueden presentar tres estructuras distintas: sustantivo (sujeto) + verbo, verbo +

\footnotetext{
${ }^{5}$ De acuerdo con Wray \& Perkins (2000, pp. 19-22) y Wray (2002, pp. 132-135), en el proceso de adquisición del lenguaje formulario en la L1 se combinan los procedimientos analítico y holístico siguiendo cuatro fases: (1) plenamente holístico en un primer momento; (2) paulatinamente más analítico a medida que se adquiere el léxico y la gramática; (3) a partir de los 8 años se empieza a usar "atajos" que permiten fusionar secuencias que antes se procesaban analíticamente; (4) al final de la adolescencia se establece un equilibrio entre ambos procedimientos, pero con preferencia por el holístico y las interpretaciones metafóricas sobre las literales. Jiang \& Nekrasova (2007) confirman la preeminencia de la perspectiva holística en el procesamiento de las secuencias formularias en una L2, lográndose mediante dos vías: integrándose en la interlengua de forma directamente holística en el caso de coincidencia entre L1 y L2, o bien a partir de un estado inicial analítico, composicional, sin conciencia de su formulaicidad, pero llegando a ser representada holísticamente gracias a su frecuencia de uso.
} 
sustantivo (objeto) $)^{6}$ y verbo + preposición + sustantivo. Además, la ausencia o presencia de determinantes (especialmente, artículos) se considera una variable a tener en cuenta, de modo que dicho sustantivo puede ser, de hecho, un sintagma nominal. En realidad, al hilo de lo expuesto por Penadés Martínez (2001, p. 68), nos parece de vital importancia esta aclaración, ya que precisamente la presencia o ausencia de determinación es una de las peculiaridades del español y puede convertirse en uno de los mayores retos de los aprendices cuya lengua materna se comporta de otra manera (especialmente, cuando carece de artículos); esta singularidad da relevancia a construcciones que no siempre podrían calificarse estrictamente como colocaciones (p. ej. comer carne, preparar la cena), pero que tienen una alta frecuencia de coaparición debido a las relaciones semánticas entre los colocados y que los discentes tienen que aprehender de forma holística en un momento del aprendizaje en el que la idiosincrasia del uso o ausencia de artículo todavía no está a su alcance? ${ }^{7}$.

Más allá de su estructura interna, estas construcciones verbo-nominales pueden clasificarse de acuerdo con criterios semántico-pragmáticos:

(1) Funcionales: formadas por un verbo soporte, es decir, un verbo que actúa como auxiliar del sustantivo, que es el que constituye realmente el núcleo semántico del sintagma; el verbo acostumbra a estar muy desemantizado, de modo que puede considerarse una proforma (hacer ruido, tomar el sol, dar un golpe, tener razón), aunque también incluiríamos en esta categoría construcciones en las que el verbo esté menos gastado semánticamente, pero que puede sustituirse por otro más general (levantar un muro vs. hacer un muro).

(2) Aspectuales: el verbo aporta un matiz aspectual, que puede ser incoativo (entablar una conversación, contraer una enfermedad, entrar en calor), durativo (mantener una conversación, transcurrir el tiempo, ejercer un cargo) o terminativo-perfectivo (zanjar una conversación, cortar una hemorragia, satisfacer una necesidad).

(3) Intensificadoras: se trata de construcciones que incluyen un verbo que implica una ponderación, generalmente cuantitativa (agravarse un problema, atiborrarse de pastillas, armarse de paciencia, cargarse de razón, cosechar éxitos) ${ }^{8}$.

\footnotetext{
${ }^{6}$ A pesar de esta diferenciación, no puede obviarse que a veces nos encontramos con la existencia de dobletes como armar(se) un escándalo o truncar(se) una esperanza, en los que la presencia del pronombre -reminiscencia de la antigua voz media- convierte el sintagma nominal en sujeto.

${ }^{7} \mathrm{~A}$ grandes rasgos, puede decirse que un sustantivo sin determinación representa un concepto virtual desvinculado de la realidad extralingüística, mientras que con un artículo (definido o indefinido) adquiere un valor referencial. Por ej. si comparamos escuchar música/canciones con escuchar una canción, está claro que en el segundo ejemplo el sustantivo contable canción tiene que remitir a una realidad extralingüística determinada, mientras que el sustantivo incontable música o el plural canciones no presentan esta función referencial, sino que solo remiten a la idea. Esta diferenciación determina la presencia o ausencia de artículo. Lamentablemente, no podemos abordar exhaustivamente esta cuestión en estas páginas, sino solo apuntar su importancia para el tema que nos ocupa.

${ }^{8}$ Debido a su especificidad pragmática, este tipo de construcción suele aparecer en niveles más elevados; por este motivo no se han encontrado en nuestro análisis de un manual de A1.
} 
(4) Léxicas: el verbo posee un significado específico, distinto en cada caso (tostar el sol, abrir(se) una herida, fruncir el ceño).

(5) Semánticas: el verbo y el sustantivo comparten unos semas específicos que elevan su frecuencia de coaparición (hablar una lengua, escuchar música, escribir un texto, leer un libro).

\section{CONSTRUCCIONES VERBO-NOMINALES EN UN MANUAL DE ELE}

Para ejemplificar cómo se puede abordar este tema en un manual de ELE desde los niveles iniciales, hemos optado por analizar un manual. Hemos elegido ;Genial! (Nivel A1) por tratarse de un método actual, fruto de la evolución de la aplicación del método comunicativo (por lo que ha podido beber de otros manuales ya clásicos como Gente, Prisma, etc.), y por su vocación de trabajar en espiral y de forma integrada los contenidos funcionales, gramaticales, léxicos y pragmáticos (Cruz Moya, 2017, p. 3). Este trabajo en espiral obliga a la reaparición de contenidos, lo cual implica también la repetición de construcciones anteriormente introducidas, de modo que a priori es un método que puede ayudar a consolidarlas y a facilitar la adquisición de la fluidez deseada, pero al mismo tiempo adecuándose a las situaciones y contenidos comunicativos que constituyen el objetivo último del manual.

En el manual se han localizado 506 construcciones verbo-nominales diferentes, las cuales se repiten una media de 2,39 veces (con un total de 1212 apariciones), de forma que podemos considerar que se lleva a cabo esa concepción cíclica de los contenidos. En cuanto a su frecuencia de uso, solo 255 (50,39\%) aparecen registradas en $R E D E S$, aunque hay que tener en cuenta que este diccionario combinatorio no se concibe como exhaustivo y obvia muchas de las construcciones que llamamos semánticas (Bosque, 2001b, pp. XXXIII-XXXIV).

En lo que se refiere a la adecuación a los objetivos comunicativos y léxicos del manual, encontramos que un $66,8 \%$ concuerdan con ellos, mientras que un $25,5 \%$ está constituido por construcciones que responden a cuestiones metalingüísticas propias de un manual de L2 u otras relacionadas con la tecnología, que es algo específico de este libro 9 , de modo que solo un $7,7 \%$ son construcciones que no obedecen a ninguno de estos criterios. Por tanto, se ve un trabajo consciente en la elección del input de este tipo.

También es importante tomar en consideración dónde aparecen. Las construcciones que se adecuan a alguno de los objetivos suelen introducirse en las muestras de

\footnotetext{
${ }^{9}$ Tal como se indica en la presentación (Cruz Moya, 2017, p. 3), este método integra de forma natural el uso de redes sociales, por lo que aparecen combinaciones como escuchar un podcast, cargarse una página web o visitar un canal, que no siempre serían esperables en otro manual de este nivel.
} 
lengua -textos, audiciones o vídeos- $(43,4 \%)$ o en el cuerpo de los ejercicios planteados $(26,6 \%)$. Por ende, podemos deducir que estas construcciones se presentan de forma integrada en los textos modelo y se practican adecuadamente en los ejercicios. En cambio, como era de esperar, las construcciones que responden a usos metalingüísticos o específicos del manual se localizan casi exclusivamente en los enunciados de los ejercicios $(84,8 \%)$ y, a mucha distancia, en las explicaciones teóricas $(6,6 \%)$. Por otro lado, las construcciones que no se adecuan a los contenidos aparecen especialmente en los enunciados $(41,7 \%)$ y en las muestras de lengua $(39,6 \%)$, lo cual puede comprenderse por el hecho de que estos textos, especialmente en los segundos, a veces aparece input adicional que escapa de los objetivos.

En lo que atañe al tipo de construcción, hemos localizado 159 semánticas y 187 léxicas diferentes, pero su índice de repetición en el manual no es equivalente, ya que las primeras se repiten una media de 2,70 veces, mientras que las segundas solo 1,97 . Por ello, en conjunto las construcciones que más aparecen son las semánticas $(35,4 \%)$, si bien seguidas de cerca por las léxicas $(30,5 \%)$. La mayor presencia de las semánticas tiene que ver con el uso de algunos verbos como comer, beber, hablar, escribir, leer, escuchar, mirar, ver, bailar o jugar a, los cuales se combinan con clases léxicas muy concretas y que pueden agruparse bajo hiperónimos (comida, bebida, idioma, texto, imagen, baile, juego o deporte). Como decíamos, este tipo de construcción no suele incluirse dentro del concepto colocación, pero se revelan esenciales a la hora de introducir ítems léxicos que se adecuen a determinadas funciones comunicativas propias del nivel A1: por ejemplo, "hablar de la comida" (comer carne/pescado/marisco/ verdura/fruta/un bocadillo/una ensalada, beber cafélleche/un refresco ${ }^{10}$ ) o "hablar de aficiones y actividades de ocio" (leer libros/cómics/un libro/un cómic/una revistal el periódico, escribir poesía/un poema, escuchar música/ una canción/la radio/un programa, ver una película/una serie/un capitulo/la televisión, mirar un vídeo/vídeos/ el Facebook, bailar tango, jugar a juegos/a un juego/a la consola/al póker/al fútbol), al tiempo que son esenciales en construcciones metalingüísticas necesarias (leer un texto/un artículo, escuchar una conversación/un diálogo).

En cuanto a las léxicas, suelen ajustarse a objetivos del manual como "describir el físico de una persona" (tener los ojos X, tener el pelo X, tener la boca X, tener un tatuaje, tener barba/perilla/rastas, llevar barba/gafas), "describir la ropa" (llevar ropa, llevar un vestido/una falda, combinar un vestido con X), "describir relaciones personales" (tener marido/esposa/pareja/amigos/un amante), "hablar de actividades cotidianas" (ir al trabajo/al colegio/a la oficina/a clase/a casa, irse a la cama), "hablar de actividades de tiempo libre" (quedar/salir con amigos, quedarse en casa, salir de fiesta, ir a una fiestala la montaña/al campo/a la playa/a la piscina/a un concierto/

${ }^{10}$ Como hemos mencionado, el tratamiento holístico de estas construcciones permite interiorizar usos gramaticales como la ausencia/presencia de artículo en relación, en este caso, con la combinación con sustantivos incontables o contables, así como percibir diferencias en el uso de sintagmas con o sin determinación como comer sopa y comer(se) una sopa. 
al gimnasio/al cine, estudiar una lengua ${ }^{11}$, tocar un instrumento/la guitarra/el piano), "organizar un viaje" (preparar un viaje, pedir información, vivir una aventura, reservar hotel, recorrer una ciudad).

Proporcionalmente, tanto en números absolutos como en índice de repetición el tercer grupo lo constituyen las funcionales $(21,2 \%)$. En estas construcciones el verbo aparece más desemantizado que en las anteriores, de manera que a menudo pueden tener equivalentes monoléxicos ( . ej. dar un paseo $=$ pasear, ir de viaje $=$ viajar $)$. Las funciones comunicativas con las que pueden asociarse concuerdan con las descritas en los casos anteriores: "hablar de actividades cotidianas" (preparar la comida/la cena, tener clase, hacer un curso, hacer la compra, dormir la siesta, tener hambre), "hablar de actividades de tiempo libre" (ir de fiesta/excursión/viaje/vacaciones/paseo/ pesca/compras, hacer un viaje/una ruta/deportelejercicio/gimnasia/fotos, practicar deporte, practicar un idioma, dar un paseo/una vuelta, hacer planes), "describir relaciones personales" (tener una relación, tener un problema), pero también otros como "dar información personal" (tener X años, tener trabajo) o "describir el tiempo atmosférico" (hacer buen tiempo/mal tiempo/frío/calor/viento/sol).

El tipo que menos aparece es el de las construcciones aspectuales (12,9\%), lo cual era esperable debido el nivel inicial del manual. Entre los verbos que dan sentido durativo tenemos estar (a dieta/en forma/de fiesta/de vacaciones/de viaje/de moda), pasar (tiempolel díalunos días) o seguir (una dieta); entre los incoativos destacan crear (un grupo/un perfil/tendencia), ponerse (unos pantalones/una camisa) u otros relacionados con la tecnología (conectarse a internet, salir un capitulo). Las terminativas o perfectivas suelen estar relacionadas con el metalenguaje del libro (completar un cuadro/una frase/una ficha), pero también con otros objetivos diversos (rellenar un formulario/un perfil, quitarse las gafas, encontrar pareja, cumplir años, salir del trabajo).

\section{CONSIDERACIONES FINALES}

Como hemos visto, estas construcciones verbo-nominales son esenciales en la interfaz léxico-sintaxis, aparecen desde el primer nivel de enseñanza y abarcan un fenómeno más inclusivo que las colocaciones. Además, muchas de ellas no solo se enmarcan dentro de campos semánticos, sino que se corresponden con funciones y situaciones comunicativas determinadas que articulan los manuales y la enseñanza de ELE.

Por ello, sería conveniente inventariar estas construcciones según su pertinencia a determinadas funciones comunicativas, adjudicándolas a un nivel concreto de ense-

\footnotetext{
${ }^{11}$ Una construcción como esta, que a ojos nativos puede parecer poco institucionalizada, en realidad se revela como fuente de confusión en lenguas en las que, como el polaco, los verbos equivalentes a estudiar y aprender tienen un comportamiento lingüístico diferente.
} 
ñanza, con lo que tanto los autores de materiales como el profesorado de ELE podría contar con una base que les permitiría introducirlas de forma consciente, gracias a lo cual se favorecería su adquisición. En realidad, es precisamente su composicionalidad semántica y su adecuación a las reglas de la sintaxis libre lo que hace que pasen desapercibidos matices de estas construcciones como la presencia o ausencia de determinación. Es evidente que para los aprendices cuya lengua materna dispone de artículos, su adquisición es más fácil (a pesar de que los usos gramaticales y pragmáticos de los artículos difieren de una lengua a otra), pero para aquellos cuya lengua carece de ellos, el valor gramatical y pragmático de los artículos escapa a su comprensión en ese primer nivel de enseñanza en el que ya debe utilizarlos.

En consecuencia, creemos que entender y tratar estas construcciones desde una perspectiva holística puede ayudar a fijarlas de forma más sólida, inculcando inconscientemente las especificidades de los usos de los artículos y, con el tiempo, contribuyendo a la interiorización de los diversos valores que pueden tener. 


\section{BIBLIOGRAFÍA}

Bosque, I. (2001a). Sobre el concepto de ‘colocación’ y sus límites. Lingüística Española Actual, 23 (1), 9-40.

Bosque, I. (Dir.) (2001b). REDES. Diccionario combinatorio del español contemporáneo. Madrid: SM.

Coseriu, E. (1991). Las solidaridades léxicas. In Principios de semántica estructural (pp. 143-161). Madrid: Gredos.

Cruz Moya, O. (Coord.) (2017). ¡Genial! Curso de español. Nivel A1. Madrid: enClave-ELE.

Dante Hernández, A.B. (2003). El componente fraseológico en la enseñanza de ELE. In M. Rodríguez (Coord.), FORMA. Formación de formadores. 6. Léxico, fraseología y falsos amigos (pp. 65-92). Madrid: SGEL.

Forment, M. (1998a). La didáctica de la fraseología ayer y hoy: del aprendizaje memorístico al agrupamiento en los repertorios de funciones comunicativas. In F. Moreno Fernández, M. Gil Bürmann \& K. Alonso (Eds.), Actas del VIII Congreso Internacional de ASELE (pp. 339-347). Alcalá de Henares: Universidad de Alcalá.

Forment, M. (1998b). La fraseología metafórica en la didáctica de segundas lenguas (emparentadas o no con la L1). Rilce, 14 (2), 225-241.

García Muruais, M.T. (1998). Propuestas para la enseñanza de unidades fraseológicas en la clase de E/LE. In F. Moreno Fernández, M. Gil Bürmann \& K. Alonso (Eds.), Actas del VIII Congreso Internacional de ASELE (pp. 363-369). Alcalá de Henares: Universidad de Alcalá.

Goldberg, A. (2006). Constructions at Work. The Nature of Generalization in Language. New York: Oxford University Press.

Higueras García, M. (1997). Las unidades léxicas y la enseñanza del léxico a extranjeros. Revista de estudios de adquisición de la lengua española, 8, 35-49.

Higueras García, M. (2006). Estudio de las colocaciones léxicas y su enseñanza en español como lengua extranjera. Málaga: Ministerio de Educación y Ciencia.

Howarth, P. (1998a). Phraseology and Second Language Proficiency. Applied Linguistics, 19 (1), 24-44.

Howarth, P. (1998b). The Phraseology of Learners' Academic Writing. In A.P. Cowie (Ed.), Phraseology. Theory, Analysis, and Applications (pp. 161-186). Oxford-New York: Oxford University Press.

Jiang, N., Nekrasova, T.M. (2007). The Processing of Formulaic Sequences by Second Language Speakers. The Modern Language Journal, 91 (3), 433-445.

Navarro, C. (2003). Didáctica de las unidades fraseológicas. In M.V. Calvi \& F. San Vicente (Eds.), Didáctica del léxico y nuevas tecnologías (pp. 99-115). Viareggio: Mauro Baroni.

Pawley, A., Syder, F.H. (2000). The One Clause at a Time Hypothesis. In H. Riggenbach (Ed.), Perspectives on Fluency (pp. 167-191). Ann Arbor: University of Michigan Press.

Penadés Martínez, I. (2001). ¿Colocaciones o locuciones verbales? Lingüistica española actual, 23 (1), 57-88.

Ruiz Campillo, J.P., Roldán Vendrell, M. (1993). Consideraciones sobre el tratamiento de las expresiones idiomáticas verbales en el aula de E/LE. In P. Barros García et al. (Eds.), Jornadas sobre aspectos de la enseñanza del español como lengua extranjera (pp. 157-165). Granada: Universidad de Granada.

Ruiz Gurillo, L. (2000). Un enfoque didáctico de la fraseología española para extranjeros. In M.J. Coperías et al. (Eds.), Aprendizaje y enseñanza de una segunda lengua (pp. 259-275). Valencia: Universitat de València.

Solano Rodríguez, M.A. (2007). El papel de la conciencia fraseológica en la enseñanza y aprendizaje de una lengua extranjera. In I. González Rey (Ed.), Les expressions figées en didactique des langues étrangères (pp. 201-211). Cortil-Wodon: EME.

Wray, A. (2002). Formulaic Language and the Lexicon. Cambridge: Cambridge University Press.

Wray, A., Perkins, M. (2000). The Functions of Formulaic Language: An Integrated Model. Language and Communication, 20 (1), 1-28. 